\title{
Análise de correlações genéricas de deflexões na restauração rodoviária e o impacto nos investimentos públicos
}

A malha rodoviária sob administração federal perfaz extensão superior a sessenta mil quilômetros, cuja restauração tem como principal parâmetro de projeto a medida de deflexão recuperável na superfície do pavimento existente, para a qual existem diversos equipamentos disponíveis para a execução dos levantamentos de campo, em especial o FWD e a Viga benkelman. Todavia os métodos de dimensionamento do reforço do pavimento normatizados pelo Departamento Nacional de Infraestrutura de Transportes (DNIT) preconizam a utilização das medidas de deflexão obtidas utilizando Viga benkelman, necessitando, portanto, de correlação da medida de deflexão obtida por outros equipamentos. Para esta correlação, existem diversas equações propostas, entretanto trata-se de equações genéricas que não consideram características específicas e condições ambientais que estão sujeitos o pavimento. Neste sentido este estudo busca apresentar a influência da utilização de equações genéricas para correlação destas medidas, demonstrando por meio de uma análise comparativa técnica a divergência entre soluções de restauração do pavimento dimensionadas através de medidas de deflexões genuínas e medidas correlacionadas, analisando inclusive a influência da correlação na aplicação de recursos públicos em manutenção rodoviária, demonstrando o prejuízo ao erário causado pelo emprego de premissas genéricas.

Palavras-chave: Pavimentação; Custo Rodoviário; Restauração Rodoviária.

\section{Analysis of generic correlations of deflections in road recover and the impact on public investments}

\begin{abstract}
The road network under federal administration extends over sixty thousand kilometers, whose restoration has as its main design parameter the measure of recoverable deflection on the existing pavement surface, for which there are various equipment available for the execution of field surveys, especially the FWD and the benkelman beam. However, the pavement reinforcement sizing methods standardized by the National Department of Transport Infrastructure (DNIT) recommend the use of deflection measurements obtained using benkelman beam, thus requiring correlation of the deflection measurement obtained by other equipment. For this correlation, there are several proposed equations, however these are generic equations that do not consider specific characteristics and environmental conditions that are subject to the pavement. In this sense, this study seeks to present the influence of the use of generic equations to correlate these measurements, demonstrating by means of a technical comparative analysis the divergence between pavement restoration solutions dimensioned through genuine deflection measurements and correlated measurements, including analyzing the influence of correlation in the application of public resources in road maintenance, demonstrating the damage to the budget caused by the use of generic premises.
\end{abstract}

Keywords: Paving; Road Cost; Road Recover.

Topic: Engenharia Civil

Reviewed anonymously in the process of blind peer.
Received: 12/08/2019

Approved: 22/11/2019
Gledson Diórgenes Santos Castro (iD)

Faculdade em Porto Nacional, Brasil

http://lattes.cnpq.br/5304788433154375

http://orcid.org/0000-0003-2822-2935

gledson.castro@dnit.gov.br

Antônio de Almeida Veras Neto (10

Universidade Federal de Goiás, Brasil

http://lattes.cnpq.br/8512716285793358

http://orcid.org/0000-0002-0358-3833

antonio.veras@dnit.gov.br

Luiz Felipe da Silva da Fonseca (iD

Universidade Federal do Rio de Janeiro, Brasi

http://lattes.cnpq.br/0309991843341444

http://orcid.org/0000-0001-8649-6702

felipe@atp-etel.com.br

\section{Rosilene Alves Lopes \\ Universidade Federal do Rio de Janeiro, Brasil \\ http://lattes.cnpq.br/4109958611581907 \\ http://orcid.org/0000-0001-6299-9372 \\ rosilopes@coc.ufri.br}

Douglas Freitas Augusto dos Santos (id

Instituto Tocantinense Presidente Antônio Carlos Porto, Brasil

http://lattes.cnpq.br/6181125172616043

http://orcid.org/0000-0001-9306-5660

eng.prof.santos@gmail.com
Referencing this:

CASTRO, G. D. S.; VERAS NETO, A. A.; FONSECA, L. F. S.; LOPES, R. A.; SANTOS, D. F. A.. Análise de correlações genéricas de deflexões na restauração rodoviária e o impacto nos investimentos públicos. Engineering Sciences, v.7, n.3, p.53-63, 2019. DOI: http://doi.org/10.6008/CBPC2318-3055.2019.003.0005 


\section{INTRODUÇÃO}

As rodovias pavimentadas sob jurisdição federal perfazem extensão superior a cinquenta mil quilômetros, sendo um grande desafio mantê-las em condições de trafegabilidade segura, considerando as condições que se encontram expostas, a exemplo de veículos com excesso de carga e condições ambientais severas. A manutenção em rodovias geralmente é contemplada através da celebração de contratos de execução de serviços de manutenção e conservação rotineira e de emergência, ou por meio de contratos de restauração rodoviária, neste segundo contemplando soluções funcionais e estruturais de revitalização do pavimento, inclusive existem modalidades de contratação que integram os dois serviços em um mesmo contrato.

Entre os anos de 2008 e 2013 foram elaborados projetos de engenharia contemplando programa de restauração e manutenção de rodovias em aproximadamente quarenta mil quilômetros de rodovias federais, que representaram aproximadamente projetos para contratação de aproximadamente 20 bilhões de reais em obras de restauração e manutenção.

\section{METODOLOGIA}

\section{Projeto de Restauração de Pavimentos Asfálticos}

\section{Premissas adotadas em Projetos de Restauração de Pavimentos Asfálticos}

Os projetos de engenharia supracitados contemplando a restauração e a manutenção do pavimento existente, indicavam prazo para execução do projeto muito reduzido visando agilizar a contratação das obras, entretanto na maioria dos casos o prazo determinado para elaboração dos estudos e projetos necessários apresentavam-se exíguos, levando as projetistas adotar soluções que aumentassem a produção de seus levantamentos de campo e elaboração de estudos e projetos.

Os métodos de dimensionamento de reforço do pavimento existentes, utilizam como principal parâmetro a medida de deflexão recuperável da superfície do revestimento, que pode ser obtida com uma série de equipamentos, sendo os mais comuns, a Viga benkelman e o Falling Weight Deflectometer, mais conhecido como FWD. Por garantir uma produção aproximadamente quatro vezes maior em relação a Viga Benkelman, para empreendimentos cujo prazo se apresente exíguo, ou para rodovias com maior tráfego, é comum a utilização do FWD para os levantamentos de medidas deflectométricas.

Entretanto apesar de apresentar maior produtividade, o FWD tem custo referente ao equipamento aproximadamente cem vezes maior que uma Viga benkelman, além de apresentar medidas de deflexão que necessitam de correlação para medidas obtidas em Viga benkelman, uma vez que os principais métodos de dimensionamento de reforço do pavimento, sendo estes o Método DNER-PRO 011/79 e o Método DNERPRO 269/94, determinam que as medidas de deflexão utilizadas no dimensionamento devem ser obtidas com emprego de Viga benkelman ou correlacionadas. 


\section{Métodos de Dimensionamento}

Atualmente existem normatizados quatro métodos de dimensionamento de espessuras de reforço de pavimentos asfálticos, sendo estes DNER-PRO 10/79 (Procedimento A - Califórnia), DNER-PRO 11/79 (Procedimento B - Argentina), DNER-PRO 159/85 e DNER-PRO 269/94. Em seguida, de forma resumida, estão apresentados os métodos baseados nos apontamentos de Fonseca (2013).

Cumpre ressaltar que os dois primeiros métodos mencionados, DNER-PRO 10/79 e DNER-PRO 11/79, são baseados no cálculo do reforço do pavimento por meio de medidas deflectométricas, sendo o primeiro método de autoria de Armando Martins Pereira, baseado no método de projeto de reforço utilizado na Califórnia - EUA e o segundo através de trabalhos realizado pelo engenheiro Bolivar Lobo Carneiro, com base nos estudos do engenheiro argentino Celestino Ruiz. Em sequência, na década de 80 foi normatizado o procedimento DNER-PRO 159/85, este baseado em avaliação de desempenho e aspectos de gerência de pavimentos, baseando-se em curvas de desempenho obtidas em rodovias brasileiras.

Na mesma época os engenheiros Ernesto Preussler e Salomão Pinto elaboraram um novo método para projeto de reforço de pavimentos flexíveis empregando critérios e conceitos advindos do método mecanístico como a consideração do Módulo de Resiliência dos Materiais. Este método foi aprimorado ao final da década de 80 e normatizado pelo DNER em 1994, como DNER-PRO 269/94, também conhecida como Método TECNAPAV. Segundo Fonseca (2013), os métodos mencionados analisam parâmetros estruturais e funcionais, sendo utilizados para o dimensionamento, critérios para cálculo da espessura de reforço estrutural de pavimentos existentes, visando o restabelecimento de sua capacidade estrutural para um novo período de projeto.

Ressalta-se que dos quatro métodos mencionados anteriormente, são geralmente utilizados por projetistas a DNER-PRO 11/79 e DNER-PRO 269/94. Em alguns termos de referência que norteiam a contratação de elaboração de projetos de engenharia para execução de obras de restauração, fica estabelecido que o projetista deverá dimensionar pelos dois métodos, comparar soluções, adotar uma delas, justificando as premissas adotadas sob o aspecto técnico e financeiro.

\section{Parâmetros de Dimensionamento}

Concentrando a análise nos dois métodos de dimensionamento de reforço de pavimento mais empregados atualmente, DNER-PRO 11/79 e DNER-PRO 269/94, verifica-se que os parâmetros necessários ao dimensionamento são: o Número 'N', que representa as solicitações provocadas pelo tráfego e é utilizado para cálculo da deflexão admissível da camada avaliada; a Deflexão Recuperável, que representa a deformabilidade das camadas do pavimento quando solicitadas a um carregamento padrão sob determinada condição; a classificação do subleito em função do Índice de Suporte Califórnia associado ao percentual de silte na parcela fina do material constituinte do subleito, sendo que esta classificação do subleito se aplica somente no método DNER-PRO 269/94. Dentre os parâmetros mencionados, verifica-se que o principal entre eles se refere a Deflexão, método não destrutivo para avaliação estrutural do pavimento, que para sua 
obtenção é necessário o emprego de equipamentos específicos para os levantamentos de campo.

\section{Deflexões Recuperáveis}

Deflexões recuperáveis, ou deformações recuperáveis, refere-se a um parâmetro de deformabilidade do pavimento, sendo o principal objeto de análise da uma avaliação estrutural. Segundo Fonseca (2013):

As deformações recuperáveis são aquelas que ocorrem ainda no regime elástico do material, ou seja, os deslocamentos verticais causados pelo carregamento do pavimento são recuperados no ato do descarregamento, retornando à condição inicial da estrutura.

Para obtenção das medidas de deflexões recuperáveis, existem diversos equipamentos que realizam tais medidas. Dentre eles destaca-se aqueles que dependem da aplicação de carga para medição da deformação provocada no pavimento, sendo alguns manuais, como a Viga benkelman e o Ensaio de Placa, e outros automatizados, como o Deflectógrafo digital Solotest, Califórnia Travelling Deflectometer e o deflectógrafo La Croix, e aqueles dinâmicos que dependem de impulso ou impacto e leitura por geofones, sensores ou LVDTs, como o Falling Weight Deflectometer (FWD) dos tipos Dynatest e Kuab.

É fato que durante muitos anos, em especial em rodovias com menor volume de tráfego, o emprego da viga benkelman era padrão nos levantamentos de medidas de deflexão, seja pela operação simples, mas também pelo baixo custo de aquisição do equipamento, em torno de cinco mil reais em valores atuais. Este método, entretanto, possui produtividade baixa em relação a outros equipamentos, variando de 5 a 10 quilômetros de faixa rodoviária por dia, sendo realizadas medidas em estações a cada 40 metros. Esta produtividade varia de acordo com condições climáticas, volume de tráfego da rodovia, topografia do segmento e qualificação da equipe de campo, portanto quanto maior a adversidade destes fatores, menor a produção diária obtida.

Ao passo que equipamentos dinâmicos por impacto, como FWD, podem atingir produtividade muito superior a atingida com emprego da Viga benkelman, o que representa uma vantagem no seu uso. Segundo DNIT (2006), ainda que existam alguns equipamentos do tipo FWD no Brasil, o seu uso não é generalizado devido ao alto custo para aquisição e utilização, além da mão de obra para operação do equipamento requerer qualificação e treinamento específico, o que representa algumas desvantagens no seu uso. Ressalta-se que os métodos de dimensionamento de reforço do pavimento utilizam as medidas de deflexão obtidas por meio do uso da viga benkelman, portanto medidas obtidas através do emprego de equipamento de tipos diferentes da viga benkelman, requerem a aplicação de equações de correlação.

\section{Equações de Correlação de Deflexões}

Conforme supramencionado, os métodos de dimensionamento de reforço de pavimento, utilizam como parâmetro de entrada medidas de deflexão obtidos com emprego de viga benkelman. Desta forma, segundo DNIT (2006), para correlação entre medidas de deflexão existem diversas equações indicadas em vários artigos, teses, dissertações e publicações, por diversos autores, a exemplo Pinto (1991) e Fabrício et al. (1994), entretanto o Manual de Restauração de Pavimentos Asfálticos (DNIT, 2006) define: "Os projetistas que utilizarem o FWD devem estabelecer suas próprias correlações com base em pesquisas locais". Outra 
fonte (DER/SP, 2006) define de forma mais clara os critérios para o segmento testemunho para definição da correlação da deflexão para um trecho específico:

Pode ser utilizada a equação de correlação entre valores obtidos pelo FWD e pela viga Benkelman, publicada na literatura técnica para levantamentos na fase de projeto básico ou de projetos executivos de pequena extensão ou obras de porte considerado pequeno. No entanto, para projetos executivos de obras de grande extensão e importância deve ser estabelecida uma equação de correlação entre as medidas de deflexões obtidas com o FWD e a viga Benkelman em segmento de, no mínimo, $1 \mathrm{~km}$ de extensão.

Justifica-se o fato da divergência da aplicação de equações genéricas para correlação entre medidas de deflexão FWD e Benkelman, pois segundo Fonseca (2013), "A não observância de diferenças de condições climáticas, constituição das estruturas do pavimento, modo de carregamento e método de ensaio empregado, podem acarretar elevados desvios".

No entanto, na contramão da boa prática da engenharia rodoviária, o principal órgão responsável pela construção e manutenção rodoviária no Brasil, o DNIT, entre os anos de 2008 e 2013, indicou nos termos de referências, anexos a editais de licitação para contratação de projetos básicos e executivos para execução de obras de restauração e manutenção rodoviária, numa extensão superior a quarenta mil quilômetros, a aplicação da equação de correlação proposta por Fabrício et al. (1994), sendo estas:

Para deflexões $D_{F W D}<85 \times 10^{-2} \mathrm{~mm}$ :

$$
D_{B}=20,645\left(D_{F W D}-19\right)^{0,351}
$$

Para deflexões $\mathrm{D}_{\mathrm{FWD}}>85$ x 10-2 mm:

$$
D_{B}=8,964\left(D_{F W D}-60\right)^{0,715}
$$

Onde:

$\mathrm{D}_{\mathrm{FWD}}=$ deflexão obtida com o FWD

$D_{B}=$ deflexão obtida com a viga Benkelman (DNIT, 2006).

Ressalta-se que os editais de licitação referentes a contratação de elaboração dos projetos supracitados, foram distribuídos em lotes de aproximadamente quinhentos quilômetros de extensão, sendo indicado nos editais em questão o prazo contratual de aproximadamente 180 dias. Este prazo se apresenta exíguo mediante os levantamentos de campo necessários, em especial as medidas de deflexão. Por este motivo, em sua larga maioria, os projetistas utilizaram o FWD para as medidas de deflexão nos segmentos rodoviários em questão, devido à alta produtividade do equipamento em relação à viga.

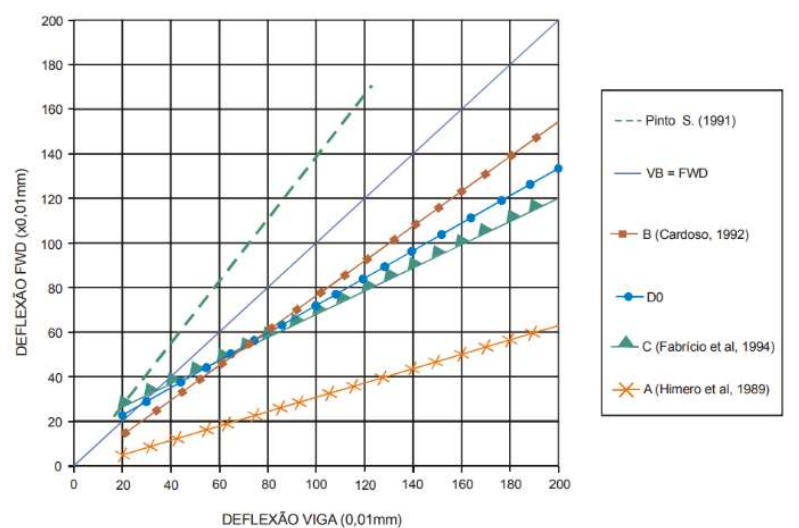

Figura 1: Correlações obtidas por vários pesquisadores (tipo DFWD = aDVB + b). Fonte: DNIT (2006).

Seguindo a determinação do termo de referência, anexo ao edital de licitação que norteou a 
contratação, as medidas de deflexão obtidas pelo FWD foram correlacionadas às medidas da Viga Benkelman por meio da equação de correlação proposta por Fabrício et al. (1994). O Manual de Restauração de Pavimentos Asfálticos do DNIT (2006), demonstra por meio de um gráfico a divergência entre resultados obtidos por estas equações de correlação, conforme ilustrado na Figura 1.

\section{DISCUSSÃO TEÓRICA}

Considerando o elevado número de projetos de engenharia referente a restauração de pavimentos asfálticos que utilizam o FWD na medida de deflexões recuperáveis, verificou-se a metodologia aplicada para correlação das medidas obtidas por FWD para medidas em Viga benkelman, sendo identificado que em larga maioria, a correlação se dava por meio de equação indicada no Manual de Restauração de Pavimentos Asfálticos do DNIT (DNIT, 2006).

Neste sentido foi identificado como estudo de caso o segmento rodoviário BR-153 situada no estado do Tocantins, entre os municípios de Aliança do Tocantins e Cariri do Tocantins. Para o segmento em questão, foi elaborado incialmente projeto de engenharia para execução de obras de restauração e manutenção, cujo dimensionamento considerou medidas de deflexão levantadas com emprego do FWD e correlacionadas por meio da equação de correlação apresentada no Manual de Restauração de Pavimentos Asfálticos do DNIT, sendo obtidas soluções de restauração ao longo do segmento e, por conseguinte um orçamento referencial para contratação das obras.

Contratadas as obras, optou-se por realizar um novo levantamento de campo, envolvendo a obtenção de medidas de deflexões recuperáveis, com o emprego direto da Viga benkelman. A partir dos resultados obtidos, foi possível realizar um novo dimensionamento do reforço do pavimento e por conseguinte um novo orçamento referencial.

Desta forma, considerando os dados obtidos, foi possível estabelecer uma análise técnica comparativa entre as medidas de deflexão obtidas por meio da Viga benkelman e as medidas obtidas por FWD e correlacionadas por equação indicada no Manual de Restauração de Pavimentos Asfálticos. Com isso foi possível estabelecer uma comparação entre as soluções indicadas com o emprego dos métodos mencionados e da análise financeira do custo envolvido para execução das soluções indicadas pelos métodos mencionados.

Através da análise comparativa supracitada, a proposta é concluir quanto ao aspecto da aplicabilidade de equações genéricas de correlação entre medidas de deflexão obtidas diretamente por Viga benkelman e medidas obtidas por FWD, em segmentos rodoviários de toda sorte, sem considerar assim suas características específicas e condições ambientais que se encontram expostos.

É fato que existem alguns estudos que anteriormente abordaram o tema, sugerindo inclusive algumas equações de correlação, entretanto este estudo busca defender a boa pratica da engenharia rodoviária em relação a adoção de equações de correlações específicas para cada segmento rodoviário, buscando justificar não somente em aspectos técnicos, mas também demonstrando financeiramente a distorção que a adoção de métodos genéricos de correlação podem apresentar, gerando a ineficiência na 
aplicação de recursos públicos na manutenção e restauração de rodovias federais. Portanto propõem-se que o DNIT adote instrução, manual ou normativo claro e objetivo quanto aos critérios para correlação de medidas de deflexões, a exemplo do Departamento de Estradas de Rodagem do estado de São Paulo.

\section{Estudo de Caso}

\section{Projeto Programa CREMA}

Para o estudo em questão, utilizamos como base de dados o Projeto Programa CREMA, elaborado em dezembro de 2017, contemplando as obras de restauração e manutenção da rodovia BR-153/TO no segmento entre o km 622,7 (Aliança do Tocantins) e km 701,1 (Cariri do Tocantins). O Projeto em questão foi elaborado por um Consórcio contratado pelo DNIT para o Gerenciamento do Programa CREMA, e norteou a contratação da execução das obras, sendo apresentado como anexo do Edital Pregão Eletrônico no 0071/2018-23 publicado em abril de 2018.

Observou-se que o Projeto em questão utilizou para medidas de deflexão o equipamento FWD, sendo utilizada a equação de Fabrício et al. (1994) para correlação com medidas viga benkelman e sua posterior aplicação no dimensionamento do reforço do pavimento existente. Seguindo o rito administrativo normal, ocorreram as fases do pregão eletrônico. A empresa Eletro Hidro Ltda foi sagrada vencedora, sendo posteriormente contratada, com ordem de início dos serviços publicada em outubro de 2018.

Em dezembro de 2018, a unidade local do DNIT em Gurupi/TO, responsável pelas rodovias federais localizadas no sul do estado do Tocantins, verificando a condição da superfície do pavimento existente e analisando as soluções indicadas no projeto para cada segmento, identificou indícios que as soluções de restauração do pavimento não se apresentavam aderentes a necessidade atual do segmento.

\section{Revisão do Projeto em Fase de Obras}

O DNIT, através da Unidade Local de Gurupi/TO, em conjunto a Superintendência do DNIT no estado do Tocantins, diante das observações da condição atual da rodovia, demandaram o consórcio supervisor responsável pela fiscalização das obras de manutenção na malha rodoviária de jurisdição da Unidade Local de Gurupi/TO, para elaboração do relatório da primeira revisão de projetos em fase de obra, para o qual demandou-se a execução de novos levantamentos de campo. Os novos levantamentos envolveram não somente a execução de medidas de deflexão com emprego da viga benkelman, mas também a avaliação objetiva da superfície do pavimento (DNIT-PRO 006/2013), além da execução de ensaios de laboratório de materiais constituintes das camadas do pavimento em segmentos que se apresentavam em piores condições.

\section{Análise das Medidas de Deflexão}

Durante a execução da Revisão de projeto em fase de obras, concluída a fase de levantamentos de campo, observou-se que as medidas de deflexão obtidas no período de revisão de projeto, realizada em dezembro de 2018, com utilização de viga benkelman, em poucos segmentos guardavam relação com as 
medidas de deflexão apresentadas no projeto original, que foram obtidas com emprego do FWD e correlacionadas com uso da equação de Fabrício et al. (1994), indicada no Manual de Restauração de Pavimentos Asfálticos (DNIT, 2006).

Comparando as medidas de deflexão obtidas pelos dois métodos, observou-se que na sua maioria, as medidas de deflexão obtidas com emprego da viga benkelman se apresentaram superiores as medidas obtidas com emprego do FWD e correlacionadas, mas como dito, não se pode afirmar como regra para o segmento em questão, pois ainda que minoria, algumas medidas com viga benkelman se apresentaram inferiores as medidas obtidas com FWD correlacionadas para o mesmo segmento rodoviário.

\section{Análise das Soluções de Restauração}

Como mencionado anteriormente, o principal parâmetro para dimensionamento da espessura de reforço do pavimento é a medida de deflexão da camada de revestimento asfáltico existente, portanto as divergências entre as medidas de deflexão obtidas com viga benkelman, em relação as medidas adotadas no projeto original, obtidas com utilização de FWD correlacionadas pela equação proposta por Fabrício et al. (1994) geraram impactos diretos nas soluções de restauração propostas pelo projeto original em sua maioria agravando as soluções de restauração do pavimento indicadas na revisão de projeto em fase de obras.

Um exemplo interessante do agravamento das soluções é facilmente identificado na extensão da solução de restauração mais intensa indicada no projeto, a reconstrução parcial do pavimento por meio da reciclagem da base existente, com incorporação do revestimento e adição de $30 \%$ de brita na sua composição, perfazendo uma camada com espessura de 20 centímetros. Sobre esta camada, faz-se a execução de Tratamento superficial duplo, com aproximadamente 2,5 centímetros espessura, em seguida a execução da camada de CBUQ faixa 'B' (Binder) como camada intermediária com espessura de 5 centímetros e por fim a execução da camada de CBUQ faixa ' $C$ ' como camada de rolamento final com espessura de 5 centímetros.

No projeto original que norteou a contratação das obras de restauração, havia previsão desta solução numa extensão de 1.750 metros de rodovia, enquanto a revisão de projeto em fase de obras, baseada nas medidas de deflexão realizadas com uso de viga benkelman, indicaram a execução da solução acima numa extensão de 20.360 metros de rodovia, perfazendo, portanto, uma extensão mais que dez vezes superior a indicada no projeto original.

\section{Análise do custo do investimento}

Diante das divergências entre as soluções de restauração do pavimento indicadas no projeto original e as soluções indicadas na revisão de projeto em fase de obras, apurou-se um acréscimo, somente nas soluções de pista, de aproximadamente 8 milhões de reais. A previsão inicial era de aproximadamente 30 milhões de reais em investimentos para recuperação da pista do segmento rodoviário em questão, passando a 38 milhões na revisão de projeto em fase de obras realizada. Esta diferença perfaz um acréscimo de $25 \%$ no custo de recuperação da pista de rolamento. 
A figura 2 apresenta um comparativo entre as medidas de deflexão obtidas à época do projeto original, com emprego do FWD, correlacionadas para medidas benkelman, e medidas obtidas à época da revisão de projetos em fase de obras, obtidas com emprego da viga benkalman. Em seguida é apresentado o quadro 01, demonstrando a cada segmento homogêneo do trecho rodoviário em questão, as soluções de restauração indicadas no projeto original, em comparação as soluções indicadas na revisão de projetos em fase de obras, além do comparativo entre o custo destas soluções de pista de rolamento.

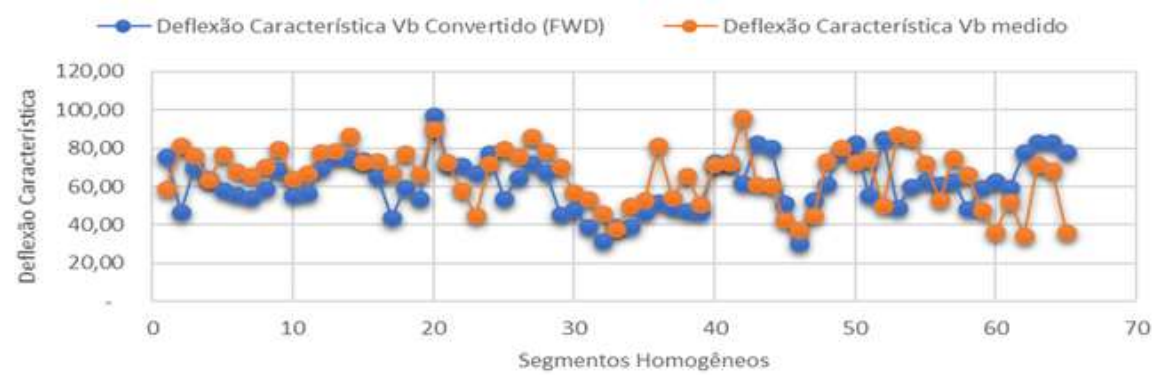

Figura 2: Gráfico Comparativo - Deflexões Convertidas x Deflexões Medidas.

Quadro 1: Quadro Comparativo de Soluções de Restauração de Pavimentação em função do Parâmetro Deflexão - (Vb convertido $\times \mathrm{Vb}$ medido).

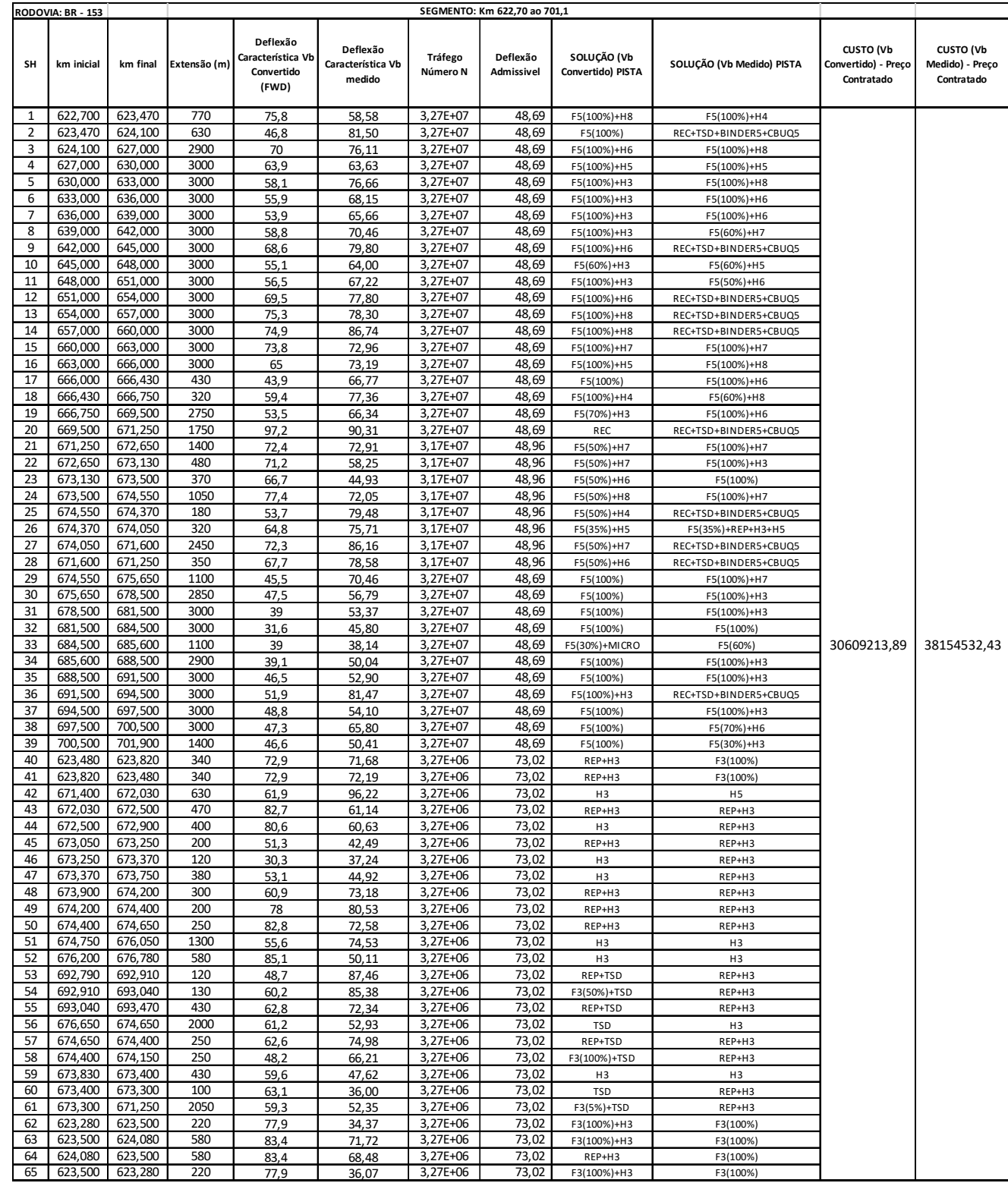




\section{CONCLUSÕES}

Diante do quadro 1 apresentado, foi possível observar os resultados obtidos no presente estudo, apresentando o impacto gerado pela aplicação de equações genéricas de correlação de medidas de deflexões. Comparando-se as medidas de deflexões medidas diretamente com viga benkelman com as medidas de deflexão obtidas com FWD e correlacionadas, não foi possível estabelecer uma correlação única para toda extensão do trecho rodoviário em estudo, portanto, é um caso claro que o trecho rodoviário em questão apresenta diversos segmentos de composição e comportamento homogêneo, sendo necessário estabelecer uma equação de correlação em cada segmento.

Quanto as soluções de restauração do pavimento existente, a indicação de soluções aquém da necessidade do segmento, que seria a consequência da execução das soluções indicadas no projeto original, representariam a execução de camadas de reforço inferiores a necessidade do segmento, face a deformabilidade do revestimento existente em relação a solicitação do carregamento do tráfego, portanto tais soluções consequentemente representariam danos precoces ao pavimento em questão e a necessidade de intervenções em período inferior ao previsto.

Como consequência das divergências apontadas nas medidas de deflexões e soluções de restauração de pavimento indicadas, estas trazem impacto direto aos investimentos financeiros empregados na execução das obras de restauração rodoviária, que no caso em questão, previam um período de 5 anos sem novas intervenções no segmento rodoviário. A execução de soluções de restauração aquém da necessidade do segmento ocasionariam a ocorrência de danos precoces ao pavimento, cujo comportamento não se apresenta linear, ou seja, a aplicação de menos recurso financeiro em função de soluções inferiores as necessárias, não traz uma redução linear da vida útil do pavimento, pois o pavimento, sujeito as solicitações do tráfego, apresentando sua estrutura protegida por um revestimento com espessura aquém da necessária, provoca danos acumulados ao revestimento com comportamento exponencial. Portanto há inconsistência em afirmar que as soluções de restauração de pavimento previstas no projeto original, cujo investimento equivale a $75 \%$ do investimento necessário indicado na revisão de projeto em fase de obras, apresentaria vida útil de $75 \%$ da vida útil projetada, ou seja, pouco mais de 3 anos.

A contribuição do presente estudo, que foi precedido por alguns estudos que objetivaram a análise de correlações de medidas de deflexão, é alertar quanto a determinação em alguns termos de referência do uso de uma equação genérica de correlação de medida de deflexão, devendo caber ao projetista seguir orientação constante no Manual de Restauração de Pavimentos Asfálticos, que sugere a adoção de correlação específica para cada segmento rodoviário. Tal procedimento tem maior detalhamento na instrução de projeto IP-DE-P00/003 do DER/SP, que estabelece uma extensão mínima para cada segmento testemunho na determinação da correlação de cada segmento específico.

Assim, buscando boas práticas na engenharia rodoviária, envolvendo o emprego de recursos públicos com eficiência, fica a contribuição deste estudo, visando despertar maior responsabilidade nos projetistas e administradores públicos, na tomada de decisão quanto as premissas a serem adotados em seus projetos e 
especificações a serem indicadas em editais de licitações.

\section{REFERÊNCIAS}

DER. Departamento de Estradas de Rodagem. Instrução de Projeto de Pavimentação IP-DE-P00/003. Instrução de Projeto. São Paulo: DER, 2006.

DNER. Departamento Nacional de Estradas de Rodagem. Norma 10/79-PRO: Procedimento A: Avaliação estrutural dos pavimentos flexíveis. MT/DNER/IPR. Rio de Janeiro: DNER, 1979.

DNER. Departamento Nacional de Estradas de Rodagem. Norma 11/79-PRO: Procedimento B: Avaliação estrutural dos pavimentos flexíveis. MT/DNER/IPR. Rio de Janeiro: DNER, 1979.

DNER. Departamento Nacional de Estradas de Rodagem. Norma ME 024/94: Método de Ensaio: Pavimento: Determinação das deflexões pela viga Benkelman. MT/DNER/IPR. Rio de Janeiro: DNER, 1994.

DNER. Departamento Nacional de Estradas de Rodagem. Norma 269/94-PRO: Procedimento: Projeto de restauração de pavimentos flexíveis: TECNAPAV (Método da Resiliência). MT/DNER/IPR. Rio de Janeiro: DNER, 1994.

DNER. Departamento Nacional de Estradas de Rodagem. Norma 273/96-PRO: Procedimento: Determinação de deflexões utilizando deflectômetro de impacto tipo "Falling Weight Deflectometer (FWD)". MT/DNER/IPR. Rio de Janeiro: DNER, 1996.

DNIT. Departamento Nacional de Infraestrutura de
Transportes. Manual de restauração de pavimentos asfálticos MT/DNIT. Brasília: DNIT, 2006.

DNIT. Departamento Nacional de Infraestrutura de Transportes. Arquivo Técnico do DNIT na SR/TO: Projeto Programa CREMA: Execução dos Serviços do programa CREMA, na rodovia BR-153/TO km 622,7 ao 701,1 MT/DNIT/SR/TO. Palmas: DNIT, 2017.

DNIT. Departamento Nacional de Infraestrutura de Transportes. Arquivo Técnico do DNIT na SR/TO: Relatório da Primeira Revisão de Projeto em Fase de Obras: Execução dos Serviços do programa CREMA, na rodovia BR-153/TO km 622,7 ao 701,1 - MT/DNIT/SR/TO. Palmas: DNIT, 2019.

FABRÍCIO, J. M.. Correlação entre Deflexões Características em Pavimentos Flexíveis Medidos com a Viga Benkelman e com o FWD - Falling Weight Deflectometer. In: REUNIÃO ANUAL DE PAVIMENTAÇÃO, 30. Anais. Salvador, 1994. p.637-646.

FONSECA, L. F. S.. Análise das Soluções de Pavimentação do Programa CREMA 2a ETAPA do Departamento Nacional de Infraestrutura de Transportes. Dissertação (Mestrado) Universidade Federal do Rio de Janeiro, Rio de Janeiro, 2013.

PINTO, S.. Estudo do comportamento à fadiga de misturas betuminosas e aplicação na avaliação estrutural de pavimentos. Tese (Doutorado) - Universidade Federal do Rio de Janeiro, Rio de Janeiro, 1991.

A CBPC - Companhia Brasileira de Produção Científica (CNPJ: 11.221.422/0001-03) detém os direitos materiais desta publicação. Os direitos referem-se à publicação do trabalho em qualquer parte do mundo, incluindo os direitos às renovações, expansões e disseminações da contribuição, bem como outros direitos subsidiários. Todos os trabalhos publicados eletronicamente poderão posteriormente ser publicados em coletâneas impressas sob coordenação da Sustenere Publishing, da Companhia Brasileira de Produção Científica e seus parceiros autorizados. Os (as) autores (as) preservam os direitos autorais, mas não têm permissão para a publicação da contribuição em outro meio, impresso ou digital, em português ou em tradução. 